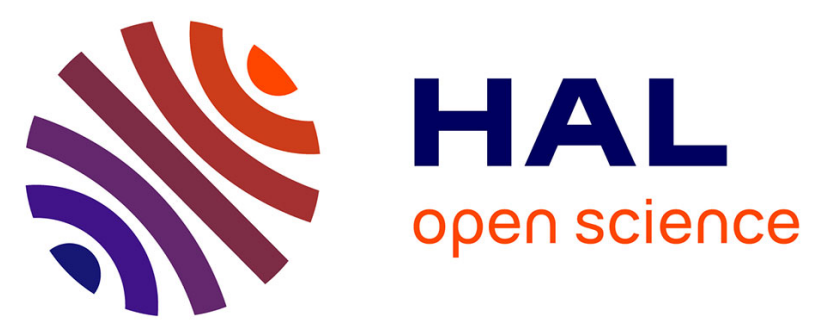

\title{
SBS Mitigation in a Microstructured Optical Fiber by Periodically Varying the Core Diameter
}

B. Stiller, Alexandre Kudlinski, M.W. Lee, G. Bouwmans, M. Delqué, J.-C.

Beugnot, H. Maillotte, T. Sylvestre

\section{- To cite this version:}

B. Stiller, Alexandre Kudlinski, M.W. Lee, G. Bouwmans, M. Delqué, et al.. SBS Mitigation in a Microstructured Optical Fiber by Periodically Varying the Core Diameter. IEEE Photonics Technology Letters, 2012, 24 (8), pp.667-669. 10.1109/LPT.2012.2186286 . hal-00707865

\section{HAL Id: hal-00707865 \\ https://hal.science/hal-00707865}

Submitted on 5 May 2021

HAL is a multi-disciplinary open access archive for the deposit and dissemination of scientific research documents, whether they are published or not. The documents may come from teaching and research institutions in France or abroad, or from public or private research centers.
L'archive ouverte pluridisciplinaire HAL, est destinée au dépôt et à la diffusion de documents scientifiques de niveau recherche, publiés ou non, émanant des établissements d'enseignement et de recherche français ou étrangers, des laboratoires publics ou privés.

\section{(c)(1)}

Distributed under a Creative Commons Attribution| 4.0 International License 


\title{
SBS Mitigation in a Microstructured Optical Fiber by Periodically Varying the Core Diameter
}

\author{
Birgit Stiller, Alexandre Kudlinski, Min Won Lee, Géraud Bouwmans, Michaël Delqué, \\ Jean-Charles Beugnot, Hervé Maillotte, and Thibaut Sylvestre
}

\begin{abstract}
In this letter, we experimentally demonstrate a 4-dB increase of the stimulated Brillouin scattering threshold in a microstructured optical fiber. This result is obtained by periodically varying the size of the air-hole structure by only 7\% amplitude, while keeping a low attenuation coefficient. The efficiency of this passive technique is verified by use of the Brillouin echoes-distributed sensing technique where the Brillouin frequency-shift oscillation is clearly observed.
\end{abstract}

Index Terms-Brillouin threshold, distributed Brillouin measurement, microstructured optical fiber, stimulated Brillouin scattering.

\section{INTRODUCTION}

$\mathbf{I}^{\mathrm{s}}$ $\mathrm{N}$ RECENT years, there have been active efforts to suppress stimulated Brillouin scattering (SBS) in optical fibers as it is detrimental to nonlinear optical processing fiber technologies and high-power narrow-linewidth fiber lasers. In addition to conventional active techniques, several passive methods have been demonstrated by applying strain or temperature distributions along the fiber [1], [2], or by fiber design [3], [4]. SBS mitigation has also been proposed using microstructured optical fibers (MOF) due to their ability to reduce the acousto-optic overlap or widen the Brillouin gain spectrum [5]. More recently, Poletti et al. suggested to increase the SBS threshold through longitudinal variations of the structural parameters of the MOF [6]. In this letter, we experimentally demonstrate $4 \mathrm{~dB}$ improvement of SBS threshold in a MOF with a periodically-varied core diameter. By varying only $7 \%$ of the air-hole microstructure over a period of $30 \mathrm{~m}$, the Brillouin frequency shift (BFS) changes significantly and the Brillouin gain spectrum (BGS) broadens. This results in $4 \mathrm{~dB}$ increase of SBS threshold, compared to a uniform fiber with an invariant core. In the following we will describe and characterize the properties of this core-diameter

B. Stiller, M. W. Lee, M. Delqué, J.-C. Beugnot, H. Maillotte, and T. Sylvestre are with the Département d'Optique, Institut FEMTO-ST, CNRS-Université de Franche-Comté, CNRS-UMR 6174, Besançon 25030, France (e-mail: birgit.stiller@femto-st.fr; minlee@femto-st.fr; michael. delque@femto-st.fr; herve.maillotte@univ-fcomte.fr; thibaut.sylvestre@ univ-fcomte.fr).

A. Kudlinski and G. Bouwmans are with the Laboratoire PhLAM, CNRSUMR 8523, IRCICA, Université Lille 1, Sciences et Technologies, Villeneuve d'Ascq 59655, France (e-mail: alexandre.kudlinski@univ-lille1.fr; geraud.bouwmans@phlam.univ-lille1.fr).

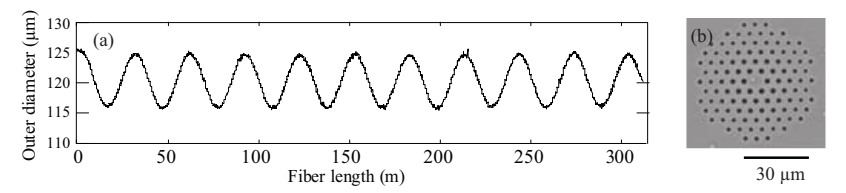

Fig. 1. (a) Outer diameter along the fiber length. (b) SEM image at the maximum outer diameter $(125 \mu \mathrm{m})$.

varied MOF by measuring the SBS threshold and the BGS linewidth. The SBS threshold is compared to the theoretical value calculated from the standard theory for a homogeneous fiber with an invariant core and to the experimental value of a MOF with a similar air-hole structure but without varying periodically the structure size. We further perform a distributed measurement of the oscillating BFS using Brillouin echoes distributed sensing (BEDS) technique. We finally estimate the corresponding effective refractive index variations from scanning electron microscopy (SEM) images and found a very good agreement with outer diameter variations.

\section{Oscillating Microstructured Optical Fiber}

The MOF with the periodically varying microstructure, shown in Fig. 1, has been drawn directly on a fiber tower. Figure 1(a) shows the evolution of its outer diameter (OD) as a function of length, measured during the drawing stage. It closely follows a sine evolution over $320 \mathrm{~m}$ with a period of $30 \mathrm{~m}$ and a modulation amplitude rate of 7\% (OD variations from 116 to $125 \mu \mathrm{m}$ ). Figure 1(b) shows a scanning electron microscope (SEM) image of the input cross-section for the largest OD. The core diameter in Fig. 1(b) is $6.4 \mu \mathrm{m}$ and the hole diameter of the two first rows around the core is $1.8 \mu \mathrm{m}$, but becomes smaller for the outer rows due to deformations during the cane drawing process. The lattice pitch $\Lambda$ is nearly constant across the whole structure and equal to $4.1 \mu \mathrm{m}$. Drawing parameters were adjusted so that OD variations only modify the pitch $\Lambda$ and keep the $d / \Lambda$ ratio approximately constant all along the fiber length. The linear attenuation of the $320 \mathrm{~m}$-long PCF sample is measured as $7.7 \mathrm{~dB} / \mathrm{km}$ at $1.55 \mu \mathrm{m}$. Using the commercial finite element method solver COMSOL, we have calculated from the SEM image zero-dispersion wavelength (ZDW) variations from $1.07 \mu \mathrm{m}$ to $1.095 \mu \mathrm{m}$. The corresponding group-velocity dispersion (GVD) parameter D at the operating wavelength of $1.55 \mu \mathrm{m}$ slightly shifts from 48.5 to $53.0 \mathrm{ps} / \mathrm{nm} / \mathrm{km}$.

\section{BRILLOUIN THRESHOLD POWER}

The Brillouin threshold has been graphically solved from Fig. 2 that shows the transmitted and backscattered powers as a 


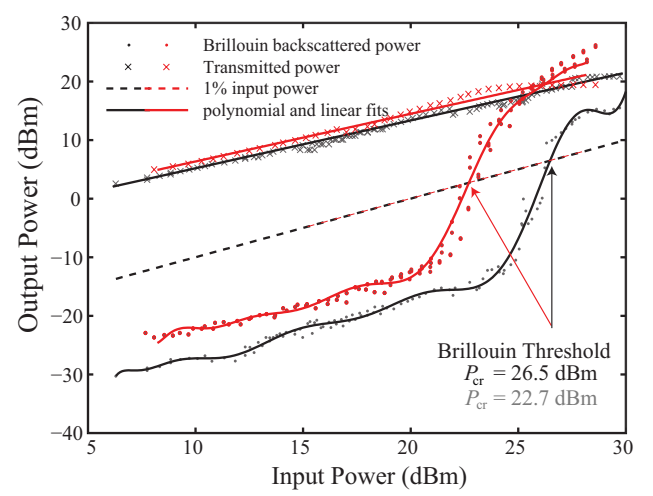

Fig. 2. Experimental measurements of the Brillouin backscattered and transmitted powers through the periodically varying MOF (black curves) and the homogenous MOF with the same microstructure but without varying structure size (red curves). The Brillouin thresholds of the two MOFs are indicated by arrows for $1 \%$ fraction of the input power.

function of the input power. In our experimental setup, we used as a pump laser a $1550 \mathrm{~nm}$ distributed feedback (DFB) fiber laser with a $45 \mathrm{kHz}$ linewidth amplified by an erbium-doped fiber amplifier (EDFA). An optical circulator was inserted at the input end of the fiber to monitor simultaneously the reflected and transmitted power. We define here the Brillouin threshold as the input power whereby the reflected power is $1 \%$ of the injected one. A polynomial fit of our experimental measurements yields the Brillouin threshold power. For the periodically varying MOF the Brillouin threshold is measured as $26.5 \mathrm{dBm}$, as shown in Fig. 2. Theoretically the Brillouin threshold for homogeneous fibers can be written as [6]:

$$
P_{\mathrm{cr}}=\frac{C \cdot K \cdot A_{\mathrm{eff}}}{g_{B} \cdot L_{\mathrm{eff}}}
$$

where $A_{\text {eff }}$ is the effective mode area, $L_{\text {eff }}$ the effective length and $\mathrm{g}_{B}$ the Brillouin gain. $\mathrm{C}$ can be expressed as $C=\psi\left(1+\frac{3 / 2 \cdot \ln (\psi)}{\psi-3 / 2}\right)$ where $\psi$ depends amongst others on the fiber parameters, the BFS, the BGS and the $1 \%$ pump power fraction [7]. $\mathrm{K}=3 / 2$ is a factor that accounts for random polarization evolution in the MOF. $\mathrm{g}_{B}$ can be calculated by measuring the full width at half maximum (FWHM) of the BGS in the spontaneous Brillouin regime [7].

Figures 3(a) and 3(b) show the BGS (FWHM) with variable input power and the BGS at $12 \mathrm{dBm}$, respectively. Measurements have been performed using a standard heterodyne detection technique where the back-scattered Brillouin signal beats with the input one [8]. As can be seen, we measure in the low-power regime $(5 \mathrm{dBm})$ an average $54 \mathrm{MHz}$ SBS linewidth (FWHM) that narrows to $10 \mathrm{MHz}$ near the threshold power $(25 \mathrm{dBm})$. This indicates a strong spectral broadening of the BGS compared to the standard value for single-mode fiber (SMF) below threshold (FWHM $\Delta v_{B}=29 \mathrm{MHz}$ ). This effect has already been reported in other MOFs as resulting from the impact of air-hole microstructure on the acoustic waves distribution [8]. It leads to a significant reduction of the Brillouin gain. Assuming an FWHM $\Delta v_{B}=54 \mathrm{MHz}$, we have estimated $\mathrm{g}_{B}=1.30 \cdot 10^{-11} \mathrm{~mW}^{-1}$. With $L_{\text {eff }}=244.2 \mathrm{~m}$, $\mathrm{C}=14.3, A_{\mathrm{eff}}=22.63 \mu \mathrm{m}^{2}$, the theoretical Brillouin threshold for a uniform silica fiber is calculated to $21.8 \mathrm{dBm}$. Thus the experimental threshold increases by more than $4 \mathrm{~dB}$
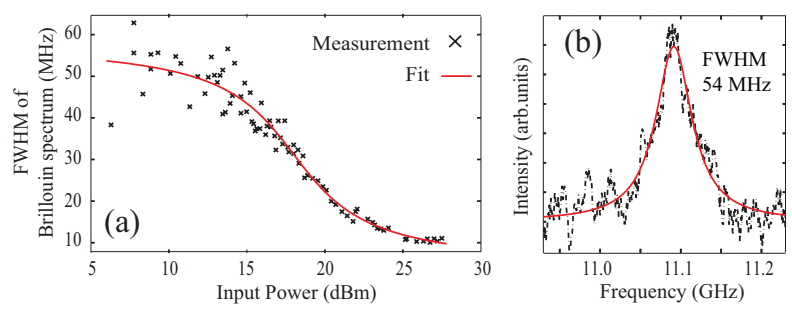

Fig. 3. (a) Brillouin gain spectrum (FWHM) with increasing input power. (b) Brillouin gain spectrum at $12-\mathrm{dBm}$ input power.

compared to this estimation. Note that this increase can be seen as a higher limit since we assumed in our calculation a uniform MOF without any periodic variation or structural irregularities that increase the Brillouin threshold [8]. Unfortunately, the model can not be used to derive a correct value in the case of periodic variation or structural irregularities. An improved theory adapted to MOFs is clearly needed.

However, to go further into detail, we have investigated a homogeneous MOF with similar air-hole microstructure. This MOF was drawn without inducing a periodic size variation. It has a length of $350 \mathrm{~m}, 4.35 \mu \mathrm{m}$ lattice pitch, $6.2 \mu \mathrm{m}$ core diameter and a mode area of $22.3 \mu \mathrm{m}^{2}$ at $1.55 \mu \mathrm{m}$. Compared to the periodically varying MOF the structural parameters are slightly different but lead to a very close effective mode area which is crucial for a fair comparison of the Brillouin threshold. The fiber attenuation is about $14 \mathrm{~dB} / \mathrm{km}$ which results in a effective fiber length of $207.6 \mathrm{~m}$. The Brillouin threshold has been measured using the same technique as for the oscillating MOF. One can see in Fig. 2 that the critical power for the Brillouin threshold (red curve) is reached at $22.7 \mathrm{dBm}$ which is almost $4 \mathrm{~dB}$ below the value for the periodically varying MOF, in good agreement with our theoretical estimation. Taking into account the important fact that the effective length of the uniform fiber is lower than for the periodically varying MOF, the Brillouin threshold can be expected to be even lower than $22.7 \mathrm{dBm}$ and the impact of the BFS variation higher than $4 \mathrm{~dB}$. Note that the FWHM of the BGS has also been measured to about $55 \mathrm{MHz}$. Thus, the periodic variations have less influence on the BGS broadening than the air-hole structure itself.

\section{Distributed Measurement}

The increase of Brillouin threshold may result from the varying BFS due to core diameter and effective refractive index variations. In order to verify this fact we have performed a distributed measurement of the BFS by use of the BEDS technique that provides a higher spatial resolution than conventional Brillouin optical time domain analysis (BOTDA) [9] without spectral broadening. The experimental setup is sketched in Fig. 4 and a detailed description can be found in Ref. [9]. In short, an amplified DFB fiber laser is split into two signals by a 50:50 tap coupler that are injected at opposite end of the fiber. One signal serves as a probe shifted by the BFS while the other one is used as a pump pulse. More precisely, a $3 n s-l o n g ~ \pi$-phase pulse is applied at the end of the pump pulse which causes a drop in the Brillouin gain 


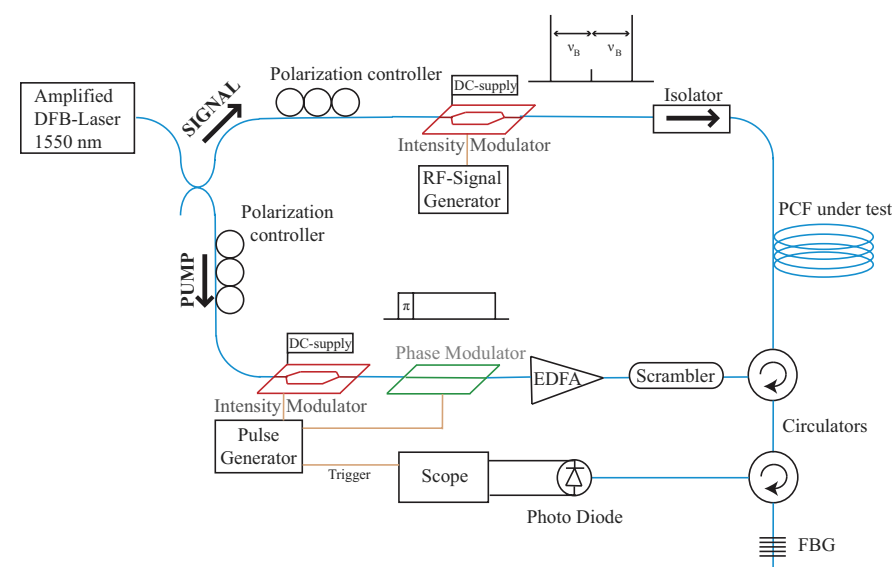

Fig. 4. Experimental setup of the Brillouin echoes distributed sensor used to measure the Brillouin frequency shift sine variations along the fiber length. DFB: distributed-feedback laser. EDFA: erbium-doped fiber amplifier. FBG: fiber Bragg grating.

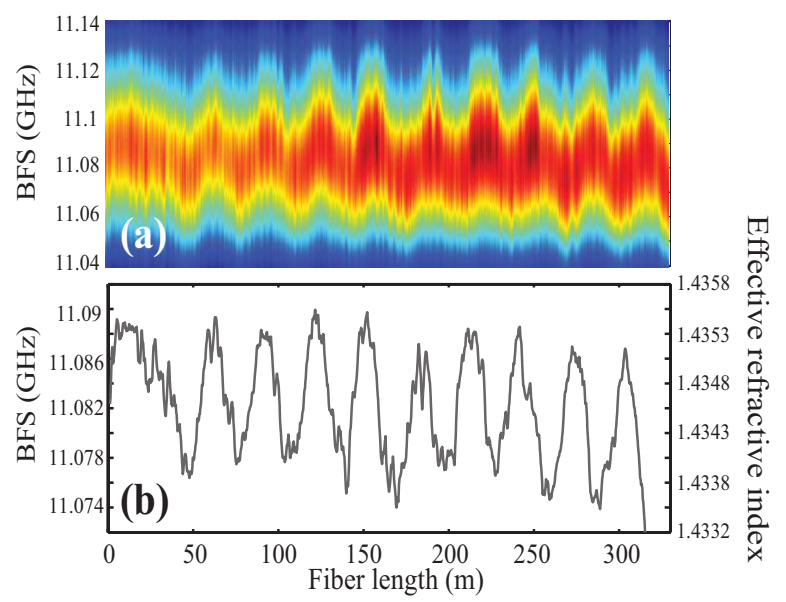

Fig. 5. Mapping of the Brillouin frequency shift along the fiber. The spatial resolution is $30 \mathrm{~cm}$ and the frequency resolution is $1 \mathrm{MHz}$. (a) Color plot of the recorded BEDS trace of the periodically varying MOF. (b) Retrieved Brillouin frequency shift variations along the fiber distance. Mean maximal BFS: $11.088 \mathrm{GHz}$; mean minimal BFS: $11.076 \mathrm{GHz}$.

detected on the counter-propagating probe. The frequency shift of the probe is obtained by an intensity modulator driven at $\mathrm{V}_{\pi}$ where two side bands shifted by the BFS are generated while suppressing the carrier wave. The output continuous-wave probe is filtered by a fiber Bragg grating (FBG) and monitored with a photodiode and an oscilloscope. The Brillouin gain is scanned by the intensity modulator around the BFS so that all variations can be detected along the fiber. The spatial resolution is set by the phase pulse duration and corresponds to $30 \mathrm{~cm}$ and the frequency resolution is $1 \mathrm{MHz}$. The input powers of the probe and pump wave are $481 \mu \mathrm{W}$ and $27 \mathrm{~mW}$, respectively.

Figures 5 (a) and (b) show a color plot of the raw BEDS trace and the retrieved BFS along the propagation distance, respectively. The periodic sinusoidal variations of the BFS can clearly be observed in these two figures. The comparison with the outer diameter variations previously shown in Fig. 1(a) is very good except in the beginning of the fiber where the first period is not observed. In addition, one can also see short-scale fluctuations of meter scale that can be attributed to structural irregularities along the fiber. In addition to the periodic variations and the BGS broadening, these short scale variations also impact on the Brillouin threshold. As the BFS is linked to the effective refractive index $n_{\text {eff }}$ by $v_{B}=\frac{2 n_{\text {eff }} V_{L}}{\lambda P}$, we can calculate the corresponding effective refractive index variations using a FEM method by scaling the fiber crosssection shown in Fig. 1(b) [10]. For the maximal and minimal sizes (corresponding to 125 and $116 \mu \mathrm{m}$ in outer diameter) of the air-hole microstructure they reveal a difference of 0.0013 in the effective refractive index. This value is consistent with the one retrieved from the distributed BFS measurement that varies from 1.4337 to 1.4353 . This confirms that the BEDS technique can provide accurate measurement of the diameter or structural variations from the BFS ones.

\section{Conclusion}

We have fabricated a microstructured optical fiber with a periodically-varying core diameter in order to mitigate the detrimental effect of stimulated Brillouin scattering. Only 7\% of outer variation enables an increase of the Brillouin threshold power by $4 \mathrm{~dB}$ compared to a homogeneous microstructured optical fiber, which is a promising result for further investigations with higher amplitude variations. The periodic oscillations of the Brillouin frequency shift has been checked by an accurate distributed measurement based on Brillouin echoes distributed sensing. Such an oscillating-core microstructured fiber may have an interesting potential for applications where Brillouin backscattering is an obstacle.

\section{REFERENCES}

[1] J. Hansryd, F. Dross, M. Westlund, P. A. Andrekson, and S. N. Knudsen, "Increase of the SBS threshold in a short highly nonlinear fiber by applying a temperature distribution," J. Lightw. Technol., vol. 19, no. 11, pp. 1691-1697, Nov. 2001.

[2] J. M. C. Boggio, J. D. Marconi, and H. L. Fragnito, "Experimental and numerical investigation of the SBS-threshold increase in an optical fiber by applying strain distributions," J. Lightw. Technol., vol. 23, no. 11, pp. 3808-3814, Nov. 2005.

[3] K. Shiraki, M. Ohashi, and M. Tateda, "Suppression of stimulated Brillouin scattering in a fibre by changing the core radius," Electron. Lett., vol. 31, no. 8, pp. 668-669, Apr. 1995.

[4] V. Laude, et al., "Phononic band-gap guidance of acoustic modes in photonic crystal fibers," Phys. Rev. B, vol. 71, no. 4, pp. 045107-1045107-6, Jan. 2005.

[5] J. Spring and B. Ward, "Brillouin gain suppression in photonic crystal fibers with random acoustically microstructured cores," Opt. Lett., vol. 35, no. 1, pp. 31-33, 2010.

[6] F. Poletti, K. Furusawa, Z. Yusoff, N. G. Broderick, and D. J. Richardson, "Nonlinear tapered holey fibers with high stimulated Brillouin scattering threshold and controlled dispersion," J. Opt. Soc. Amer. B, vol. 24, no. 9, pp. 2185-2194, 2007.

[7] A. Kobyakov, M. Sauer, and D. Chowdhury, "Stimulated Brillouin scattering in optical fibers," Adv. Opt. Photon., vol. 2, no. 1, pp. 1-59, 2010.

[8] J.-C. Beugnot, et al., "Complete experimental characterization of stimulated Brillouin scattering in photonic crystal fiber," Opt. Express, vol. 15, no. 15, pp. 15517-15522, 2007.

[9] S. M. Foaleng, M. Tur, J.-C. Beugnot, and L. Thévenaz, "High spatial and spectral resolution long-range sensing using Brillouin echoes," J. Lightw. Technol., vol. 28, no. 20, pp. 2993-3003, Oct. 15, 2010.

[10] B. Stiller, et al., "Photonic crystal fiber mapping using Brillouin echoes distributed sensing," Opt. Express, vol. 18, no. 19, pp. 20136-20142, 2010. 